\title{
Effect of Aloe vera Extract on Depression in People with Prediabetes
}

\author{
Mohsen Foadoddini (iD ${ }^{1}$ and Samaneh Alinejad Mofrad (iD) ${ }^{2,}$ \\ ${ }^{1}$ Cardiovascular Disease Research Center, Birjand University of Medical Sciences, Birjand, Iran \\ ${ }^{2}$ School of Nursing and Midwifery, Birjand University of Medical Sciences, Birjand, Iran \\ "Corresponding author: School of Nursing and Midwifery, Birjand University of Medical Sciences, Central Organization of Birjand University of Medical Sciences, Ghafari St, \\ Birjand, Iran. Tel: +98-9155053868, Email: alinejads1@yahoo.com
}

Received 2020 January 12; Revised 2020 April 11; Accepted 2020 April 25.

\begin{abstract}
Background: Depression is one of the major mood disorders that leads to development of type 2 diabetes in pre-diabetic patients . Any intervention s alleviate the symptoms of depression in these patients can significantly help preventing type 2 diabetes.

Objectives: The effects of pure extract powder of Aloe vera on depression in prediabetic patients were examined.

Methods: A double blind randomized controlled trial study was conducted on 72 prediabetic patients. After sampling the patients were allocated to three groups (Aloe vera $300 \mathrm{Mg}$ (AL300), Aloe vera $500 \mathrm{Mg}$ (AL500) and Placebo (PL). The participants in the two first groups received pure extract powder of Aloe Vera capsules twice a day in the morning and evening for eight weeks. In addition, they completed Beck Depression inventory before and eight weeks sfter the intervention. After confirming the normality of the mean depression score variable by the Kolmogorov-Smirnov test, the data was analyzed using ANOVA, Chi-square, and paired t-test. Results: After the 8th week of intervention, the mean depression scores in the $500 \mathrm{Mg}$ aloe vera (AL500) group decreased significantly $(\mathrm{P}=0.033)$.

Conclusions: Aloe vera extract powder (AL500) considerably alleviated depression mean scores in prediabetic patients during an eight weeks period.
\end{abstract}

Keywords: Aloe vera, Prediabetic State, Depression

\section{Background}

Diabetes mellitus is characterized by an increased level of blood glucose and impairments in the metabolic functions of carbohydrates, fats, and proteins (1).

There are more than 347 million people with diabetes across the world, which is expected to reach 366 million by 2030 (2).

Prediabetes state is a condition of abnormal blood glucose level between normal and diabetic states that involves impaired fasting glucose (IFG) and impaired glucose tolerance (IGT). It is a major risk factor for type 2 diabetes. The World Health Organization (WHO) considers fasting blood glucose (FBG) equivalent to $100-125 \mathrm{mg} / \mathrm{dL}$ as the prediabetic state (3).

In addition to physical problems, it is known that some psychological disorders such as stress, anxiety, sleep disorders, and daily depression are associated with an increased risk of diabetes development $(4,5)$. Depression is on the list of risk factors suggested by the American Diabetes Association for the preliminary screening of diabetes (6). The association between depression and diabetes has been discussed for years. As two serious chronic diseases, they have negative impacts on the quality of life, disability, and life expectancy (7).

Chien et al. (8) concluded in their study that the incidence of diabetes in individuals suffering from at least one year of daily depression is greater than the general population.

On the other hand, some hormones, as well as cytokines and interleukins in diabetic patients, raise the incidence of depression (5). Diabetes, together with depression, can lead to worsened glycemic index control, delayed insulin therapy, lower self-confidence, lack of energy, sedentary lifestyle, reduced self-care behaviors, and even the incidence of dementia in patients with diabetes. Unfortunately, depression is usually left unnoticed in these people, and treatment is not initiated accordingly (9).

Depression can also result in psychological-biological changes such as lack of control of the autonomic nervous system, increased levels of cortisol, and increased inflammatory markers that can collectively deteriorate glycemic index management (4).

In the last decade, the use of complementary therapies has increased to relieve various health problems (10). Of 
all the complementary treatments, herbal medical therapy has played an effective role in promoting the quality of life for many years (11).

Aloe vera is the safest and most widely used plants in different cultures. Aloe vera (Aloe vera L., Liliaceae family) has applications in health products and has antioxidant, anti-cancer, and anti-inflammatory properties (12). Some compounds of Aloe vera are effective in regulating mood such as vitamins, enzymes, minerals, Lignin, salicylic acid, and amino acids. Also, copper, iron, magnesium, arachidonic acid, Y-linolenic acid, steroids, mannose, glucose, Lrhamnose, aldopentose, vitamin A, B12, C, E, choline and folic acid, auxins, and gibberellins have been found in this plant $(12,13)$. Mood disorders such as depression can be caused by changes in neurotransmitters activity due to the impairment in brain neurons' activity. The Aloe vera plant has soothing and mood regulating properties (12).

No complications have been reported for Aloe vera consumption at different doses in the course of time $(12,14)$. In one study, the extract of this plant led to changes in neurotransmitter function and reduction in symptoms of depression in laboratory rats (15). Thus, the purpose of our research was to investigate the effects of pure extract powder of Aloe vera at two doses on depression in prediabetic patients.

\section{Methods}

This was a double-blind, randomized, controlled trial performed in the Research Center of Birjand University of Medical Sciences (BUMS). It was approved by the Ethics Committee of BUMS with the code number: IR.BUMS.REC.1394.451. Further, the trial was registered in the Iranian Registry of Clinical Trials with the number IRCT2013041112984N1. This study was performed among 72 volunteers meeting the inclusion criteria including age between 35 and 65 years, fasting blood sugar (FBS) between 100 and $125 \mathrm{mg} / \mathrm{dL}$, triglyceride (TG) between 150 and $200 \mathrm{mg} / \mathrm{dL}$, total cholesterol (CHOL-T) between 200 and $250 \mathrm{mg} / \mathrm{dL}$, high-density lipoprotein (HDL) less than $35 \mathrm{mg} / \mathrm{dL}$, low-density lipoprotein (LDL) between 130 and $160 \mathrm{mg} / \mathrm{dl}$, body mass index (BMI) between 25 and 30 (3), not experienced any serious stress history during the past two months (such as the death of one family member, losing a job or anything that was very horrible and affected the person's routine), no history of type 2 diabetes, gestational diabetes and other chronic diseases, not using antidepression drugs, no pregnancy, no breastfeeding, not having hepatic, renal or gastrointestinal diseases according to medical examination, and not having elective surgery in the past two months. The exclusion criteria included a lack of willingness to continue participation, not using the capsules for more than one week, and pregnancy during the study.

None of the participants knew the contents of the capsules. To determine the sample size, we used the formula for comparing the difference between two means in independent groups (power calculation $=80 \%$ and confidence $=95 \%$ ) based on Devaraj et al. (16) study results. According to purposive non-probability sampling, the block randomization method was used for the random allocation of the patients to the three groups $(n=24$; Figure 1 ). The AL500 group consumed 500-mg capsules of Aloe vera (Barij Essence Company, Iran), the AL300 group received $300 \mathrm{mg}$ capsules of Aloe vera and the placebo group received capsules containing microcrystalline cellulose powder. All the groups received two capsules per day, one after breakfast and one after dinner for eight weeks.

To prepare the extract powder of Aloe vera in Barij Essence Company, at first, freshly harvested whole Aloe vera leaves were washed in a suitable bactericide (chlorhexidine). Then, 1 inch of the leaf base and 2 inches of the tapering point and sharp spines located along the leaf margins were removed by a knife. Then, the skin was carefully separated from the parenchyma. The filets were extensively washed with distilled water to remove the exudates from their surfaces, and the filets were ground to a liquid, and the pulp was removed by filtering. Afterwards, the pure extract of Aloe vera was extracted and then powdered.

The study objectives were explained to volunteers as clearly and simply as possible, and their informed consent was obtained. Then, a fasting blood sample (10 cc) was taken to confirm that the patient is a prediabetic. After taking a blood sample, the needle head was removed, and the blood was slowly transferred to a simple tube. This sample was kept at room temperature for 20 minutes and then centrifuged for 10 - 15 minutes to separate the serum. Serum variables were measured by a chemistry analyzer (Prestige 24i, Japan) using biochemical kits (ParsAzmoon, Iran) in the Central Laboratory of Imam Reza Hospital of Birjand.

Beck depression inventory was completed by the patients for calculating the depression mean scores before the study. Then, the package of the capsules with the timeline of how to use them was given to the participants.

The participants visited the Research Center to complete the questionnaire at the end of the eighth week of treatment. The researcher's phone number was also given to the participants for probable queries. In addition, all the participants were reminded to take the pills and were emphasized not to use any other herbal medicines. Finally, they were requested not to have any change in their diet 


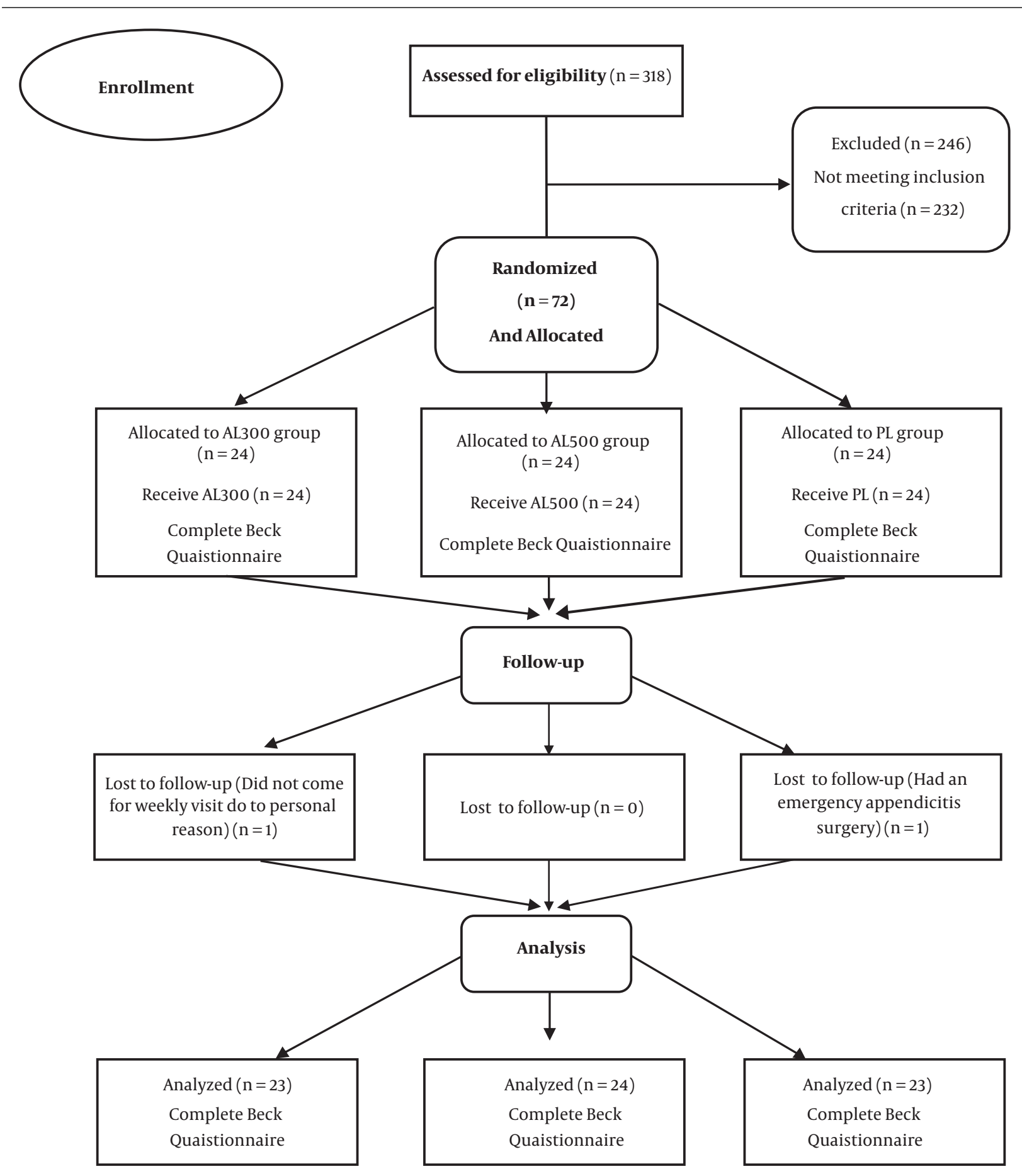

Figure 1. Flowchart of the project procedure and attrition in the groups

and routine activities during the study period.

The data were analyzed in SPSS (version 16), and the results are described as mean \pm SD (standard deviation). A paired $t$-test was used to determine any significant differ- ence between the groups, and analysis of variance (ANOVA) followed by Tukey's post-hoc test was run to determine inter-group differences. The significance level was set at $\mathrm{P}$ $\leq 0.05$. 


\section{Results}

The demographics of the participants and the parameters investigated are presented in Table 1. Chi-square and ANOVA showed the homogeneity of the demographic characteristics. No adverse events were reported during the study period. The capsules were well-tolerated, and no complaints were reported about their taste or smell.

Table 2 shows a significant reduction in depression mean score only in the group receiving 500-mg capsules of Aloe vera $(\mathrm{P}=0.034)$. Also, depression mean score changes showed a significant difference in depression mean score in the group receiving 500-mg capsules of Aloe vera) AL 500) compared with the two other groups (AL300 and placebo; $\mathrm{P}=0.033$ )

In addition, results of the Tukey's post-hoc test showed that depression mean score changes before and 60 days after intervention were different in the 300 and 500-mg Aloe vera groups $(\mathrm{P}=0.046)$, that is, depression decreased more in the 500-mg group than the 300-mg group.

\section{Discussion}

There are many psychological factors contributing to accelerating the progression of type 2 diabetes. Daily depression is the most important psychological factor involved in diabetes development (5). The results showed that only 500-mg Aloe vera capsules could significantly reduce the mean depression score 60 days after the intervention. But, the 300-mg Aloe vera capsule did not have a significant effect on improving the mean depression score. The reason for this finding could be that probably medicinal plants at lower doses may need a longer time to show their therapeutic effects. On the other hand, mood disorders such as depression require longer periods or higher doses of the drug to show signs of improvement (17). In fact, this is the first human study to investigate the effect of Aloe vera extract on depression. Previous studies have evaluated its effect on depression in laboratory animals. Salehi et al. investigated the antidepressant effects of Aloe vera extract compared to fluoxetine on rats. The rats were divided into five groups and given Aloe vera extract and fluoxetine for two weeks. The results showed that Aloe vera extract at different doses showed good and acceptable antidepressant effects $(\mathrm{P}<0.001$ (compared to fluoxetine ( $\mathrm{P}$ $=0.1)(18)$.

Another study examined the effect of Aloe vera on memory and depression in laboratory rats. The rats were divided into two groups, one in the depressed group and the other in the group with reduced memory. The results showed that Aloe vera extract had no effects on memory improvement in rats but significantly reduced their depression $(\mathrm{P}<0.05)(19)$.

The results of a cross-sectional study showed that men and women with symptoms of daily depression, albeit mild depression, present a significantly higher prevalence of metabolic syndrome. They also reported that women with daily depression have higher levels of fasting blood sugar than healthy women (20). The reason for the incidence of diabetes following daily depression is that the body of a depressed person secretes a large amount of cortisol, which can reduce insulin levels, leading to increased resistance against it. In general, mood disorders such as depression can be caused by changes in neurotransmitters' activity arisen from impaired function of brain neurons (5). In fact, Aloe vera has soothing and mood-regulating properties (21). Thus, the present study investigated the impact of Aloe vera on depression. Further studies are required to clearly establish its role as an antidepressant. We had some limitations in control of some confounding variables such as finding the prediabetic patients and determining food intake, which could be considered for future researches.

\subsection{Conclusions}

Pure extract powder of Aloe vera in the AL500 group could considerably reduce depression mean scores in prediabetic patients after eight weeks of treatment.

\section{Acknowledgments}

Sincere thanks go to all who helped us in conducting this study.

\section{Footnotes}

Authors' Contribution: Study concept and design: SAM and MF. Analysis and interpretation of data and drafting of the manuscript: SAM. Critical revision of the manuscript for important intellectual content: SAM and MF. Statistical analysis: SAM.

Clinical Trial Registration Code: The clinical trial registration code was IRCT2013041112984N1.

Conflict of Interests: The authors do not have any conflicts of interest.

Ethical Approval: The ethical code was IR.BUMS.Rec.1394.451.

Funding/Support: This research has been funded by Birjand University of Medical Sciences.

Informed Consent: The study objectives were explained to the volunteers as clearly and simply as possible and then their informed consent was obtained. 


\begin{tabular}{|c|c|c|c|c|}
\hline Variable & PL & AL300 & AL500 & P Value \\
\hline Age, $y$ & $54.5 \pm 7.7$ & $49.5 \pm 7.5$ & $53.2 \pm 9.9$ & 0.14 \\
\hline Man & $6(26.1)$ & $5(21.7)$ & $10(41.7)$ & $0.29^{\mathrm{b}}$ \\
\hline Woman & $17(73.9)$ & $18(78.3)$ & $14(58.3)$ & \\
\hline The mean depression score & $12.56 \pm 1.56$ & $13.47 \pm 2.15$ & $13.3 \pm 2.51$ & 0.37 \\
\hline HbA1C & $6 \pm 0.16$ & $6 \pm 0.24$ & $6 \pm 0.23$ & 0.37 \\
\hline FBS, $\mathrm{mg} / \mathrm{dL}$ & $110.1 \pm 3.9$ & $112.2 \pm 2.5$ & $111 \pm 4.1$ & 0.149 \\
\hline
\end{tabular}

Abbreviations: AL300, Aloe vera 300-mg capsule group; AL500, Aloe vera 500-mg capsule group; Pl, placebo group.

${ }^{a}$ Values are expressed as No. (\%) or mean \pm SD.

${ }^{\mathrm{b}}$ Chi-square test was applied to check homogeneity of the groups in terms of gender and other variables, ANOVA was used.

\begin{tabular}{|c|c|c|c|c|c|}
\hline \multirow{3}{*}{ Variables (Time) } & \multicolumn{5}{|c|}{ Groups } \\
\hline & \multicolumn{3}{|c|}{ Mean Depression Score } & \multicolumn{2}{|c|}{ Mean Depression Score Changes } \\
\hline & Befor the Intervention & After 8 Weeks & Paired $t$-test Results & $\begin{array}{l}\text { Before the Intervention } \\
\text { to } 8 \text { Weeks After That }\end{array}$ & ANOVA Test Results \\
\hline Aloe vera, $500 \mathrm{mg}$ & $13.3 \pm 2.51$ & $11.7 \pm 2$ & $0.034^{*}$ & $-1.6 \pm 1.17$ & \multirow{3}{*}{$\begin{array}{c}\mathrm{P}=0.033^{*} \text { Tukey post-hoc } \\
\text { test }=0.046\end{array}$} \\
\hline Aloe vera, $300 \mathrm{mg}$ & $13.47 \pm 2.15$ & $13.12 \pm 1.99$ & 0.18 & $0.35 \pm 0.91-$ & \\
\hline Placebo & $12.56 \pm 1.56$ & $12.73 \pm 1.68$ & 0.51 & $0.17 \pm 1.26-$ & \\
\hline
\end{tabular}

${ }^{\mathrm{a}}$ Values are expressed as mean $\pm \mathrm{SD}$.

${ }^{b}$ All P values listed refer to the comparison between the study onset and eighth week of intervention in the same group.

${ }^{\mathrm{C}} \mathrm{P}<0.05$ in comparison as four times corresponding in the control group.

\section{References}

1. Papatheodorou K, Banach M, Bekiari E, Rizzo M, Edmonds M. Complications of diabetes 2017. Journal of Diabetes Research. 2018;2018. doi: $10.1155 / 2018 / 3086167$.

2. Cho NH1, Shaw JE, Karuranga S, Huang Y, da Rocha Fernandes JD, Ohlrogge AW, et al. IDF Diabetes Atlas: Global estimates of diabetes prevalence for 2017 and projections for 2045. Diabetes research and clinical practice. 2018;138:271-81.

3. Kasper DL, Fauci AS, Hauser SL, Longo DL, Jameson JL, Loscalzo J. Harrison's Principles of Internal Medicine, (Vol. 1 \& Vol. 2). United States: McGraw Hill Professional; 2018.

4. Lloyd CE, Nouwen A, Sartorius N, Ahmed HU, Alvarez A, Bahendeka $S$, et al. Prevalence and correlates of depressive disorders in people with Type 2 diabetes: results from the International Prevalence and Treatment of Diabetes and Depression (INTERPRET-DD) study, a collaborative study carried out in 14 countries. Diabetic Medicine. 2018;35(6):760-9.

5. Sartorius N. Depression and diabetes. Dialogues in clinical neuroscience. 2018;20(1):47.

6. Dias LS, Nienov OH, Goelzer Neto CF, Schmid H. Unsteady walking as a symptom in type 2 diabetes mellitus: independent association with depression and sedentary lifestyle and no association with diabetic neuropathy. Brazilian Journal of Medical and Biological Research. 2018;51(5).

7. Teshome HM, Ayalew GD, Shiferaw FW, Leshargie CT, Boneya DJ. The prevalence of depression among diabetic patients in Ethiopia: a systematic review and meta-analysis, 2018. Depression research and treatment. 2018;2018. doi: 10.1155/2018/6135460.

8. Chien I, Wu E, Lin C, Chou Y, Chou P. Prevalence of diabetes in patients with major depressive disorder: a population-based study. Comprehensive psychiatry. 2012;53(5):569-75.
9. Candar A, Demirci H, Baran AK, Akpınar Y. The association between quality of life and complementary and alternative medicine use in patients with diabetes mellitus. Complementary therapies in clinical practice. 2018;31:1-6.

10. Micozzi MS. Fundamentals of Complementary, Alternative, and Integrative Medicine-E-Book. USA: Elsevier Health Sciences; 2018.

11. Maqbool M, Dar MA, Gani I, Mir SA, Khan M. Herbal medicines as an alternative source of therapy: A review. World J Pharm Pharm Sci. 2018;80(2):374-80.

12. Shakib Z, Shahraki N, Razavi BM, Hosseinzadeh H. Aloe vera as an herbal medicine in the treatment of metabolic syndrome: A review. Phytotherapy Research. 2019;23(10):2649-60.

13. Oveissi V, Bahramsoltani R, Farzaei MH, Rahimi R. Effects of Aloe vera (L.) Burm. f. in gingivitis: A review of clinical trials. Research Journal of Pharmacognosy. 2017;4(Supplement):57.

14. Alinejad-Mofrad S, Foadoddini M, Saadatjoo SA, Shayesteh M. Improvement of glucose and lipid profile status with Aloe vera in prediabetic subjects: A randomized controlled-trial. Journal of Diabetes $\&$ Metabolic Disorders. 2015;14(1):22.

15. Tabatabaei SRF, Ghaderi S, Bahrami-Tapehebur M, Farbood Y, Rashno M. Aloe vera gel improves behavioral deficits and oxidative status in streptozotocin-induced diabetic rats. Biomedicine \& Pharmacotherapy. 2017;96:279-90.

16. Devaraj S, Yimam M, Brownell LA, Jialal I, Singh S, Jia Q. Effects of Aloe vera supplementation in subjects with prediabetes/metabolic syndrome. Metabolic syndrome and related disorders. 2013;11(1):35-40.

17. Gershon A, Thompson R. Mood disorders. General Psychology FA18. 2018:155.

18. Salehi B, Biazar E, Jahromi Hadipour M, Romani Akbari H. Antidepressant effects of aloe vera hydro alcoholic extract on mice model. Journal of Paramedical Sciences (Jps). 2011;2(3):59-63. 
19. Halder S, Mehta AK, Mediratta PK. Aloe vera improves memory and reduces depression in mice. Nutritional neuroscience. 2013;16(6):2504 .

20. Raatikainen I, Mäntyselkä P, Vanhala M, Heinonen A, Koponen H, Kautiainen $\mathrm{H}$, et al. Leisure time physical activity and its relation to psychiatric comorbidities in depression. Findings from Finnish Depres- sion and Metabolic Syndrome in Adults (FDMSA) study. Journal of affective disorders. 2019;259:150-3.

21. Asokkumar K, Ramachandran S. Herb-Drug Interactions: Focus on Adverse Drug Reactions and Pharmacovigilance of Herbal Medicines. Herbal Medicine in India. Germany: Springer; 2020. p. 547-71. doi: 10.1007/978-981-13-7248-3_33. 